\title{
Novel instrumentation for spectrally resolved soft x-ray plasma tomography: Development and pilot results on TEXTOR
}

\author{
L. A. Shmaenok ${ }^{a}$ \\ Phystex, Dukatenburg 30b, 3437 AC, Nieuwegein, The Netherlands and A. F. Ioffe Physical-Technical \\ Institute, Polytechnicheskaya 26, 194021 St. Petersburg, Russia
}

S. V. Golovkin, V. N. Govorun, and A. V. Ekimov

Institute for High Energy Physics, 142284 Protvino, Moscow region, Russia

N. N. Salashchenko

Institute for Physics of Microstructures, Ulyanova 46, 603600 Nizhny Novgorod, Russia

V. V. Pickalov

Institute of Theoretical and Applied Mechanics, Institutskaya 4/1, 63009 Novosibirsk, Russia

V. P. Belik

A. F. Ioffe Physical-Technical Institute, Polytechnicheskaya 26, 194021 St. Petersburg, Russia

F. C. Schüller, A. J. H. Donné, and A. A. M. Oomens

FOM Institute for Plasma Physics, EURATOM-FOM Association, Partner in the Trilateral Euregio Cluster, P.O. 1207, Nieuwegein, The Netherlands

K. A. Prokhorov and S. S. Andreev

Institute for Physics of Microstructures, Ulyanova 46, 603600 Nizhny Novgorod, Russia

A. A. Sorokin, B. G. Podlaskin, and L. V. Khasanov

A. F. Ioffe Physical-Technical Institute, Polytechnicheskaya 26, 194021 St. Petersburg, Russia

(Presented on 22 June 2000)

\begin{abstract}
A novel instrumentation for wavelength- and time-resolved plasma emission tomography in the range 0.1-4 keV has been demonstrated on the Torus Experiment for Technology Oriented Research (TEXTOR). The technique is intended for reconstruction of distributions of local emission coefficients (LEC) for selected spectral lines of impurity ions. Further determination (with additional data on electron density and temperature) of spatial distributions of impurity ions at particular ionization stages will become feasible. Spectrally selective plasma images at several viewpoints around plasma are obtained with miniature pinhole cameras supplemented with multilayer mirrors as dispersion elements. The x-ray image is converted to a visible image and transported by a fiber bundle to a gain enhanced recording camera with an electron bombarded charge coupled device tube. A part of the system has been installed on temporary TEXTOR ports. First demonstration results have been obtained on plasma imaging and on subsequent LEC reconstruction using a modified iterative sinogram restoration tomography algorithm. The complete diagnostics will be operational after the TEXTOR shutdown in 2001. (C) 2001 American Institute of Physics. [DOI: 10.1063/1.1319372]
\end{abstract}

\section{INTRODUCTION}

Impurity transport has been recognized as a crucial process determining basic problems in fusion plasma physics. Its role has been further realized in recent years in experiments with seeded impurities (Ne, Ar): the so-called radiative improved mode associated with an essential improvement of the energy confinement was demonstrated at the Torus Experiment for Technology Oriented Research $(\text { TEXTOR })^{1}$ and later at other facilities including the Axially Symmetric Divertor Experiment Upgrade, DIII-D, and the Joint European Torus. ${ }^{2}$ The observed coupling of processes in the radiating plasma mantle and in the plasma core is a strong motivation factor for a comprehensive study of impu-

${ }^{\text {a)} E l e c t r o n i c ~ m a i l: ~ p h y s t e x @ k n o w a r e . n l ~}$ rity transport, based on detailed experimental data on impurities in the whole plasma cross section. A successful approach to this fundamental problem can be based on a tomography study of the distribution of ions in the plasma with sufficient spatial and time resolution.

Here we report on the development of a novel instrumentation for spectrally selective, time-resolved plasma emission tomography in the range $0.1-4 \mathrm{keV}$ and pilot results on application of this diagnostic on TEXTOR. The instrumentation [indicated in the list of FOM diagnostics on TEXTOR as ultrasoft $x$-ray tomography (USXT) $\left.{ }^{3}\right]$ is intended for reconstruction of distributions of local emission coefficients (LEC) for impurity ions at particular ionization stages. This is seen as the main distinctive quality as compared to the existing well developed x-ray tomography systems. ${ }^{4,5}$ In combination with additional data on density 


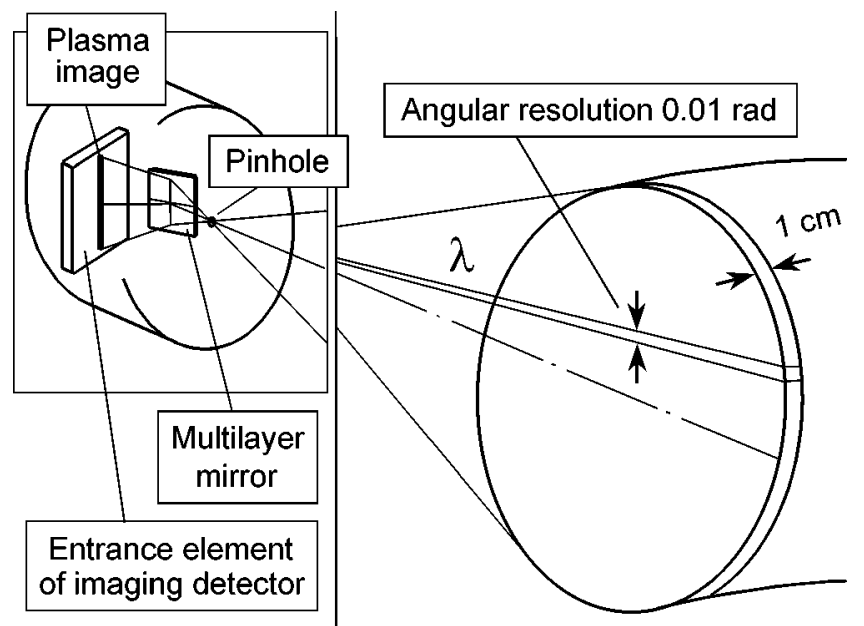

FIG. 1. Principle of spectrally selective soft x-ray plasma imaging.

and temperature distributions of plasma electrons, LEC distributions will enable reconstruction of transport processes involving specific ions.

Preliminary results on the instrumentation development were reported in Ref. 6.

\section{PRINCIPAL SOLUTION}

The novel approach is based on the use of miniature wavelength-resolving pinhole cameras, equipped with advanced soft $\mathrm{x}$-ray multilayer mirrors (MLM). As seen in Fig. 1 , one camera creates a de-magnified side image of a thin region, "sliced" across the plasma torus. A high-resolution multilayer mirror is installed at a certain angle with respect to the plasma cross-section plane, corresponding to the emission line wavelength of the selected ion. To collect this line radiation from a plane cross section, the MLM is made graded, i.e., with a certain variation of the period along the image line. The x-ray image is converted to a visible one in a phosphor layer deposited on the entrance surface of a fiber optical image conduit, transmitting the image to a remote charge coupled device (CCD)-based detector. The detector-an electron bombarded CCD (EBCCD) cameraprovides time-resolved recording of the images through the plasma discharge. Due to compactness of the MLM/pinhole camera and remote location of the EBCCD camera, a tomog- raphy system including at number of imaging cameras becomes quite feasible on a plasma facility saturated with other diagnostics. This means a drastic advantage of the new concept as compared to that of the earlier developed MLMbased multichannel monochromators. ${ }^{7}$

\section{INSTRUMENTATION PERFORMANCE}

\section{A. Essential components}

Multilayer mirrors of a high resolution ${ }^{8,9}$ provide the necessary spectral selectivity of imaging. MLMs used in this work have been optimized for emission lines of intrinsic and seeded plasma impurities in the spectral range $0.1-4 \mathrm{keV}$. Table I contains parameters of structures, fabricated for pilot tomography experiments. Most of the indicated impurity lines have already been recorded on TEXTOR using this set of mirrors.

Electron bombarded CCD cameras ${ }^{10}$ have been selected as gain enhanced imaging detectors with a high signal-tonoise ratio. In the EBCCD cameras developed for this instrumentation photoelectrons transfer (with linear demagnification of 0.52 ) the image from the photocathode to the CCD chip image zone of a $9.04 \times 6.67 \mathrm{~mm}^{2}(532 \times 290$ pixels). The photoelectrons are accelerated at a high voltage of up to $10 \mathrm{kV}$, providing in the CCD a gain of $2 \times 10^{3}$. The camera normally records the images at a $50 \mathrm{~Hz}$ rate during the whole plasma discharge. A resolution of $1 \mathrm{~ms}$ can be realized within separate $10 \mathrm{~ms}$ intervals. The camera is equipped with fast, low noise, compact electronics positioned near the EBCCD tube.

\section{B. Imaging module}

The instrumentation has been designed and fabricated as a system of independent x-ray imaging modules. Each module contains a MLM/pinhole camera equipped with sets of changeable multilayer mirrors and pinholes, a fiber image conduit, and an EBCCD camera. The MLM/pinhole camera design can be adapted to the location port. Figure 2 shows the module fabricated for mounting on TEXTOR $63 \mathrm{~mm}$ valves. Another design version enabling positioning of the MLM/pinhole assembly inside the plasma vessel during operations (to increase the view angle from $40^{\circ}$ to $60^{\circ}-80^{\circ}$ ) is in progress. Selection and positioning of MLMs and pinholes

TABLE I. Parameters of multilayer mirrors optimized for specific impurity lines.

\begin{tabular}{lcccccc}
\hline \hline \multicolumn{1}{c}{ Ion } & $\begin{array}{c}\lambda \\
(\mathrm{nm})\end{array}$ & $\begin{array}{c}\text { Multilayer } \\
\text { structure }\end{array}$ & $\begin{array}{c}\text { Period, } \\
(\mathrm{nm})\end{array}$ & $\begin{array}{c}\text { Grazing angle, } \\
(\mathrm{deg})\end{array}$ & $\begin{array}{c}\text { Reflectivity, } \\
(\%)\end{array}$ & $\begin{array}{c}\text { Resolution, } \\
\lambda / \Delta \lambda\end{array}$ \\
\hline Ar XVIII & 0.373 & $\mathrm{Cr} / \mathrm{Sc}$ & 2.0 & 5.4 & 30 & 150 \\
Ar XVII & 0.395 & $\mathrm{Cr} / \mathrm{Sc}$ & 2.0 & 5.7 & 30 & 160 \\
Ne X & 1.213 & $\mathrm{~W} / \mathrm{Si}$ & 2.1 & 17 & 13 & 96 \\
Ne IX & 1.345 & $\mathrm{~W} / \mathrm{Si}$ & 2.1 & 19 & 6 & 90 \\
O VIII & 1.90 & $\mathrm{~W} / \mathrm{Si}$ & 2.1 & 27 & 7 & 80 \\
O VII & 2.16 & $\mathrm{Cr} / \mathrm{Sc}$ & 2.1 & 31 & 7 & 130 \\
Ar XIV & 2.74 & $\mathrm{Fe} / \mathrm{Ti}$ & 2.5 & 33.6 & 9 & 140 \\
C VI & 3.37 & $\mathrm{Cr} / \mathrm{Sc}$ & 2.5 & 41 & 12 & 140 \\
C V & 4.03 & $\mathrm{Cr} / \mathrm{Sc}$ & 3.1 & 40 & 10 & 125 \\
B V & 4.86 & $\mathrm{Fe} / \mathrm{C}$ & 3.8 & 40 & 40 & 80 \\
B IV & 6.03 & $\mathrm{Cr} / \mathrm{C}$ & 4.7 & & & 75 \\
\hline \hline
\end{tabular}




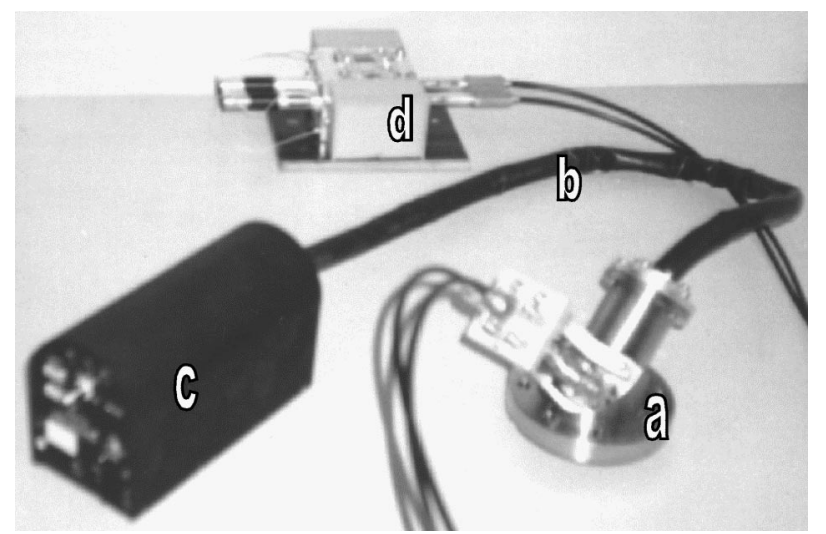

FIG. 2. Imaging module: (a) MLM/pinhole camera, (b) fiber image conduit, (c) EBCCD camera, (d) driver of MLMs and pinholes.

can be performed between plasma discharges with a driver utilizing reliable optical sensors. ${ }^{11}$ The camera is coupled to the EBCCD camera with a 1-m-long fiber conduit with a 1 $\mathrm{cm}^{2}$ entrance cross section, composed of $10 \mu \mathrm{m}$ fibers. An angular resolution of up to $0.01 \mathrm{rad}$ in the cross-section plane can be achieved corresponding to collection of radiation with one camera effectively along about 100 chords.

The imaging modules have been extensively tested and calibrated at laboratory facilities equipped with soft $\mathrm{x}$-ray sources, monochromators, and various optical accessories. The main measurements were associated with sensitivity of each module, its variation within the image field, absolute spectral and radiometric calibration. In particular, sensitivity "maps" of the imaging modules' entrance optics were measured using a laser plasma $\mathrm{x}$-ray radiation source.

\section{INSTALLATION ON TEXTOR}

The tomography system on TEXTOR will include six imaging modules, positioned in one poloidal cross section.

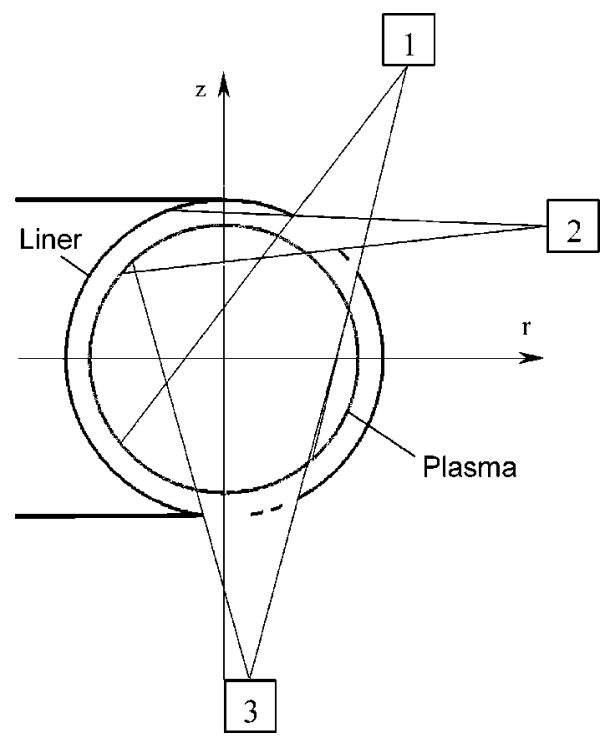

FIG. 3. Radiation collection geometry of temporary installation on TEXTOR.

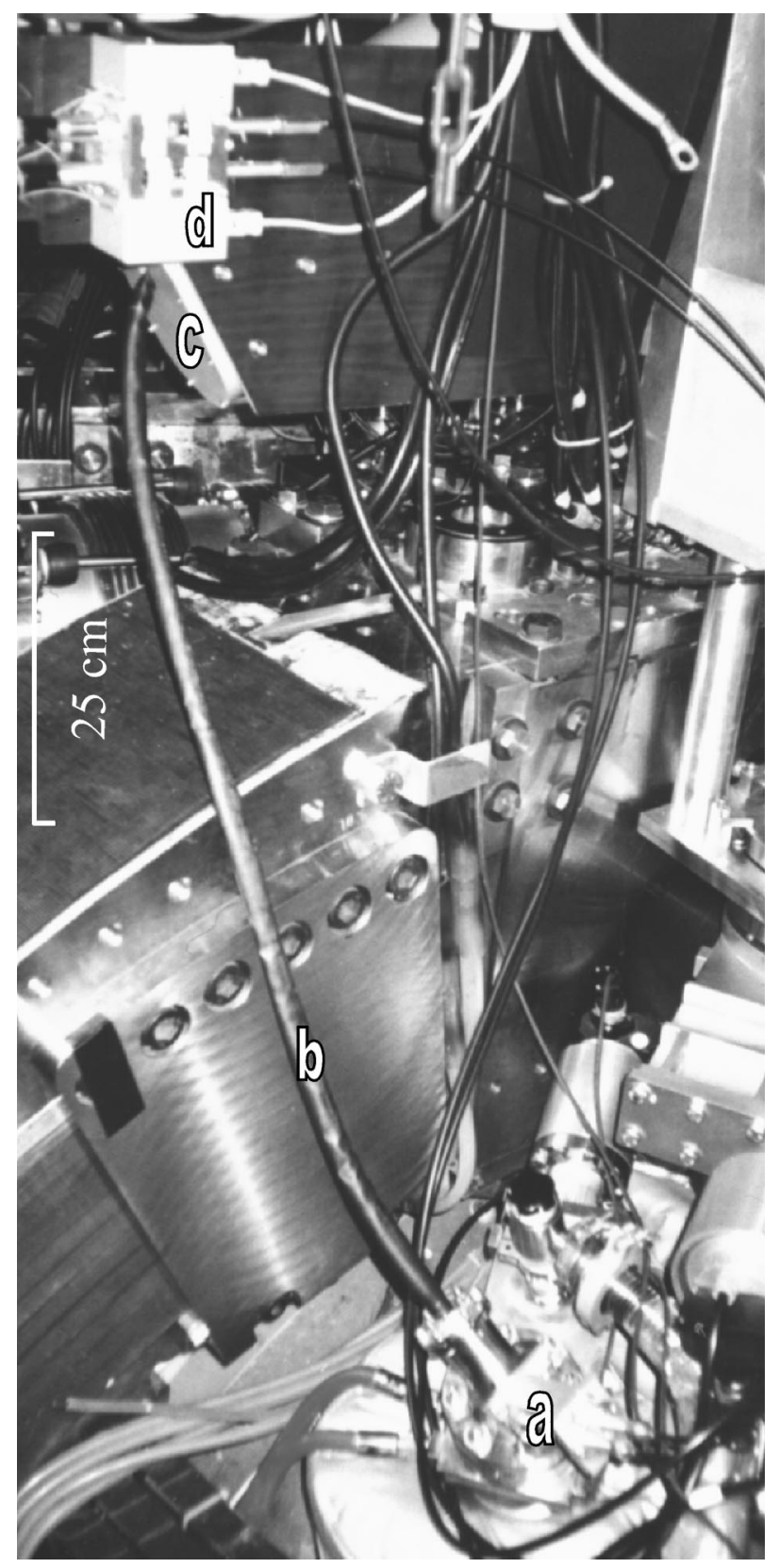

FIG. 4. One of three imaging modules (labels as in Fig. 2) currently mounted on TEXTOR. EBCCD camera (c) is inside magnetic shield.

The final installation is scheduled for 2001, after modification of the TEXTOR liner during the planned shutdown. A temporary installation of $3 \mathrm{MLM} /$ pinhole cameras - to test and demonstrate system performance using currently available ports-is shown in Fig. 3. One of the modules (upper) mounted on TEXTOR is seen in Fig. 4. The radiation collection angles are determined by the ports and the liner geometry and by construction elements of the liner. The bottom camera (3) is installed separately, in a cross section, remote from that of cameras 1 and 2 by $45^{\circ}$ of the large torus circumference. Nevertheless, in spite of these temporary limitations, important functionality tests of the system have been performed, resulting in probe reconstruction of LEC distributions for several impurity lines. 


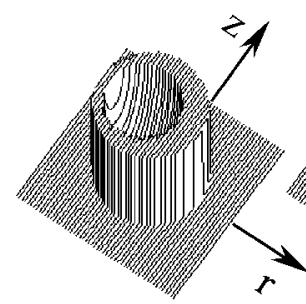

a

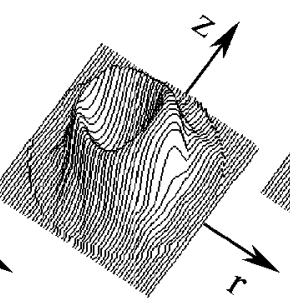

b

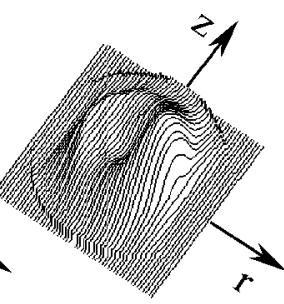

$\mathrm{c}$
FIG. 5. (a) Simulation results on reconstruction of exact model for two cases of radiation collection by three cameras, positioned at present mounting points on TEXTOR: (b) assumed full view of plasma by each camera and (c) view within real angles shown in Fig. 3.

\section{TOMOGRAPHY CALCULATIONS}

The instrumentation developments are supplemented by numerical simulations of imaging experiments using known and new tomography algorithms. ${ }^{12}$ The phantoms library includes smooth hollow profiles, additions as highly localized disturbances, models for axially symmetrical and asymmetrical distributions.

The calculation technique has been further adapted to the real installation geometry on TEXTOR due to modification of the previously developed iterative sinogram restoration (ISR) algorithm, ${ }^{13}$ which can be uniquely applied to a very irregular spatial distribution of view lines. For the current temporary layout of three cameras, which only partly cover the poloidal plasma cross section (Fig. 3), additional assumptions, such as up-down symmetry, were introduced in ISR in some calculations. Figure 5 illustrates the influence of the present angular limitations on reconstruction quality. A hollow-shaped circular model is reconstructed with an average error of $44 \%$ with the assumed full plasma view angles for each of three cameras. The average error increases to $57 \%$ for the real angles shown in Fig. 3, however the main deformation effect is seen at one side, caused by the lack of emission information from a part of the cross section (a strong-field plasma side, as seen in Fig. 3). This simulation predicted similar deformations of distributions reconstructed from experimental data (Fig. 7).

\section{PLASMA IMAGING}

Time-resolved plasma images in emission lines of $\mathrm{C} \mathrm{V}$, C VI, O VIII, Ne IX, Ne X were recorded with three imaging modules in various discharges during several experimental sessions at TEXTOR. Figure 3 shows typical image frames for lines of Ne IX $(1.35 \mathrm{~nm})$ and $\mathrm{Ne} X(1.21 \mathrm{~nm})$. Simultaneous imaging of close spectral lines like Ne IX and Ne X enabling to monitor ions at different ionization stages is a valuable advantage for impurity transport studies.

The images were processed taking into account the above-mentioned pre-installation calibration measurements of instrumental parameters, such as sensitivity maps of the imaging modules. Intensity distributions along the lines serve as initial data for time-resolved reconstruction of LEC. In the first experiments the time resolution was reduced from 20 to $100-200 \mathrm{~ms}$ because of the use of a single frame

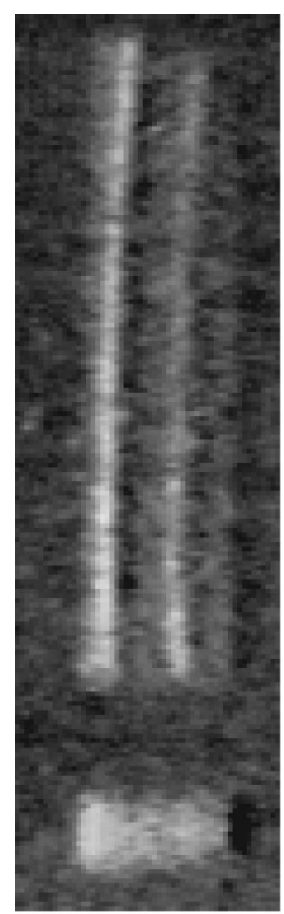

Camera 1

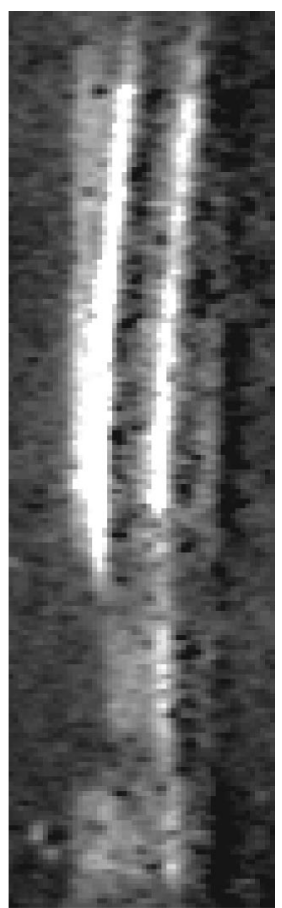

Camera 3
FIG. 6. Typical plasma images in lines of Ne IX (1.35 nm, right line) and $\mathrm{Ne} X(1.21 \mathrm{~nm}$, left line), recorded with cameras 1 and 3 (Fig. 3). Images are obscured by construction elements of TEXTOR liner (schematically shown in Fig. 3).

grabber (Matrox Pulsar) with three cameras. The specified resolution of $20 \mathrm{~ms}$ will be provided for the complete system with new data acquisition electronics.

\section{PILOT RESULTS ON TOMOGRAPHY RECONSTRUCTION}

Reconstruction results shown below in Fig. 7 were obtained using the above-mentioned modified ISR algorithm, with the same number of iterations (75) and effective chords (35 for each of cameras 1 and 3) for all sets of plasma images. Because of poor data, determined by the limited view of plasma from the temporary locations of the cameras, ad-

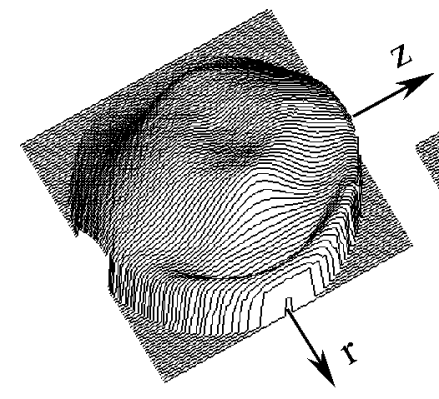

a

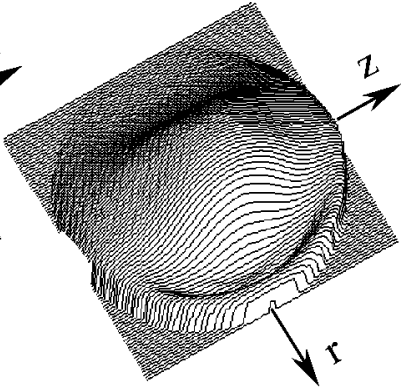

b
FIG. 7. Time-resolved reconstruction of LEC distributions for Ne IX (a, $\left.\mathrm{a}^{\prime}\right)$ and $\mathrm{Ne} X\left(b, b^{\prime}\right)$ from imaging data obtained simultaneously, as shown in Fig. 6. Essential deformation of reconstructed shapes at one side is observed, caused by absence of emission data in that area. 
ditional assumption of up-down plasma symmetry was introduced in some calculations. However, this assumption has not significantly changed the calculated distributions. For the above discussed reasons, the pilot LEC reconstructions are to be treated just as demonstration results providing an opportunity to estimate the future work after installation of the complete system.

\section{SUMMARY}

A novel instrumentation for spectrally selective, timeresolved soft $\mathrm{x}$-ray tomography of plasma impurities has been demonstrated. It includes miniature wavelengthresolving MLM/pinhole cameras coupled with EBCCD detectors, composing independent imaging modules. First three instrumentation modules have been installed and tested on temporary ports on TEXTOR. The results have proved applicability of the main concept as well as of the specific technical solutions. Time-resolved plasma images in selected emission lines of intrinsic and seeded impurities have been recorded by all cameras. The ISR tomography algorithm was adapted to the real radiation collection geometry. In spite of limitations of the current installation, important functionality tests of the system have been performed, resulting in demonstration of reconstruction of LEC distributions for several impurity lines. These pilot results are considered as the basis for future full-scale implementation of the diagnostics.

\section{ACKNOWLEDGMENTS}

This work is performed under the Euratom-FOM association agreement with financial support from Euratom and NWO. The authors appreciate support and assistance of B. S. Q. Elzendoorn, W. Kooijman, F. H. Bohn, S. V. Gaponov, S. V. Bobashev, Vl. Pikalov, N. A. Tokranova, A. Vasil'ev.

Presented at the Proceedings of the 13th Topical Conference on HighTemperature Plasma Diagnostics, Tucson, AZ, 18-22 June 2000.

${ }^{1}$ U. Samm et al., Plasma Phys. Controlled Fusion 35, B167 (1993).

${ }^{2}$ J. Ongena et al., Plasma Phys. Controlled Fusion 41, A379 (1999).

${ }^{3}$ A. J. H. Donné et al., Rev. Sci. Instrum. (these proceedings).

${ }^{4}$ M. Anton et al., Plasma Phys. Controlled Fusion 38, 1849 (1996).

${ }^{5}$ L. C. Ingesson et al., Plasma Phys. Controlled Fusion 42, 161 (1996).

${ }^{6}$ L. A. Shmaenok et al., Proc. 26th EPS Conf. Contr. Fus. and Plasma Phys., Maastricht, 1999, Vol. 23J, pp. 769-805.

${ }^{7}$ L. A. Shmaenok, Y. Y. Platonov, and N. N. Salashchenko et al., J. Electron Spectrosc. Relat. Phenom. 80, 259 (1996).

${ }^{8}$ S. S. Andreev et al., Proc. SPIE 3406, 45 (1998).

${ }^{9}$ N. N. Salashchenko et al., Mikrochim. Acta 405, 292 (1998).

${ }^{10}$ S. V. Golovkin et al., Proc. of SPIE Int. Symp. on Opt. Sci., Eng. and Instrum. San Diego, 1995, Vol. 2551.

${ }^{11}$ B. G. Podlaskin et al., Sov. Phys. Tech. Phys. 40, 805 (1995).

${ }^{12}$ V. V. Pickalov and T. S. Melnikova, Plasma Tomography. Nauka Novosibirsk (in Russian).

${ }^{13}$ L. C. Ingesson and V. V. Pickalov, J. Phys. D 29, 3009 (1996). 